\title{
Asymptotic Expansions for a Class of Elliptic Difference Schemes*
}

\author{
By Göran Starius
}

\begin{abstract}
In this paper, we derive an asymptotic expansion of the global error for Kreiss' difference scheme for the Dirichlet problem for Poisson's equation. This scheme, combined with a deferred correction procedure or the Richardson extrapolation technique, yields a method of accuracy at least $O\left(h^{6.5}\right)$ in $L_{2}$, where $h$ is the mesh length.
\end{abstract}

1. Introduction. In Section 2 of this paper we consider a family of difference schemes for the Dirichlet problem for Poisson's equation in $n$ dimensions. The schemes are based on the standard $(2 n+1)$-point formula combined with polynomial extrapolation formulas of high degree, $k$ say, at the boundary. Kreiss has developed an interesting method for proving the convergence of schemes of this kind, by reducing the stability investigations to one-dimensional problems. In a recent paper by Pereyra, Proskurowski, and Widlund [2], the stability has been proved, for $1 \leqslant k \leqslant 6$, by using Kreiss' method. In the paper [2], it is also proved that, for $k=6$, there exists an asymptotic expansion of the global error of the form

$$
v=u+h^{2} e_{2}+h^{4} e_{4}+r_{h}, \quad\left\|r_{h}\right\|_{2}=O\left(h^{5.5}\right),
$$

where $v$ and $u$ are the solutions to the discrete and the continuous problems, respectively, $h$ is the mesh length, $e_{2}$ and $e_{4}$ are smooth functions independent of $h$, and $\|\cdot\|_{2}$ is the usual discrete $n$-dimensional $L_{2}$-norm. The main result of Section 2 is the following extension of the above expansion

$$
v=u+h^{2} e_{2}+h^{4} e_{4}+h^{6} e_{6}+r_{h}, \quad\left\|r_{h}\right\|_{2}=O\left(h^{6.5}\right),
$$

which is obtained by a refined stability investigation with respect to the inhomogeneous term in the boundary condition. By using three or four different mesh lengths, (1.1) guarantees that we get an error of order $O\left(h^{6}\right)$ or $O\left(h^{6.5}\right)$, respectively, by the Richardson extrapolation method. A deferred correction method is very likely less costly to use since it only requires one mesh length; see [1]. For a description of the latter method and for several numerical experiments see [2].

Finally, we point out that the kind of meshes used in this paper are not suitable for Neumann problems, for which we instead suggest the use of composite mesh methods; see [3] and [4].

2. An Asymptotic Expansion of the Global Error for Kreiss' Method. We begin this section with a brief account of Kreiss' difference scheme for the Dirichlet problem for Poisson's equation. Almost the same notations will be used as in [2],

Received December 19, 1979; revised October 17, 1980.

1980 Mathematics Subject Classification. Primary 65B05, 65N15.

* This work was supported by the Swedish Natural Research Council (NFR 2711-018). 
where also a more thorough description of the method can be found. The continuous problem is denoted by

$$
\begin{aligned}
-\sum_{i=1}^{n} \partial^{2} u / \partial x_{i}^{2} & =f(x), \quad x \in \Omega, \\
u(x) & =g(x), \quad x \in \partial \Omega,
\end{aligned}
$$

where the region $\Omega$ is an open, bounded subset of the $n$-dimensional, real Euclidean space $R^{n}$ with the smooth boundary $\partial \Omega$. The smoothness requirements needed for the solution will be apparent later.

A uniform grid $R_{h}^{n}$ is defined by

$$
R_{h}^{n}=\left\{x \in R^{n} \mid x_{i}=x_{i}^{(0)}+n_{i} h, n_{i}=0, \pm 1, \pm 2, \ldots\right\},
$$

where $h>0$ is the mesh length and $\left(x_{1}^{(0)}, x_{2}^{(0)}, \ldots, x_{n}^{(0)}\right)$ is a fixed point in $R^{n}$. Let $\Omega_{h}=\Omega \cap R_{h}^{n}$ and define $\Omega_{h}^{*}$ to be the set of gridpoints $x \in \Omega_{h}$ such that at least one of the points $x \pm h e_{i}, i=1,2, \ldots, n$, is not in $\Omega_{h}$, where the vector $e_{i}$ is the unit vector in the direction of the positive $i$ th coordinate axis. The points in $\Omega_{h}^{*}$ are called irregular gridpoints. For each $x \in \Omega_{h}$, we initially apply the second-order difference approximation

$$
2 n v(x)-\sum_{i=1}^{n}\left(v\left(x-h e_{i}\right)+v\left(x+h e_{i}\right)\right)=h^{2} f(x) .
$$

For an irregular gridpoint $x$, this formula is modified in the following way. Assume that $x-h e_{i} \notin \Omega_{h}$. Then $v\left(x-h e_{i}\right)$ shall be eliminated from (2.2) by using a polynomial exirapolation formula of a fixed degree $k$

$$
\begin{gathered}
-v\left(x-h e_{i}\right)=\sum_{j=1}^{k} \beta_{j} v\left(x+h(j-1) e_{i}\right)-\frac{1}{\alpha_{0}} g\left(x^{*}\right), \\
\beta_{j}=(-1)^{j+1} \frac{s}{j-s}\left(\begin{array}{l}
k \\
j
\end{array}\right), \quad j=1,2, \ldots, k, \\
\alpha_{0}=(1-s)(2-s)(3-s) \cdots(k-s) / k !,
\end{gathered}
$$

where $x^{*}$ is the intersection of $\partial \Omega$ and the line segment between $x-h e_{i}$ and $x$ and hence $x^{*}=x-h e_{i}+s h e_{i}$, where $0<s<1$. It is now easily seen that the coefficient matrix $A$ of the difference scheme can be written as

$$
A=\sum_{i=1}^{n} P_{i}^{T} A_{i} P_{i}
$$

where the matrices $A_{i}$ correspond to diffrences in the $i$ th coordinate direction and are the direct sum of matrices of the form

$$
\left(\begin{array}{crrrrr}
\left(2+\beta_{1}\right) & \left(-1+\beta_{2}\right) & \beta_{3}, \ldots, \beta_{k} & & & \\
-1 & 2 & -1 \ldots & & & \\
0 & -1 & 2 & \ldots 2 & -1 & 0 \\
\cdots & & & \ldots-1 & 2 & -1 \\
& & & \tilde{\beta}_{k}, \ldots, \tilde{\beta}_{3} & \left(-1+\tilde{\beta}_{2}\right) & \left(2+\tilde{\beta}_{1}\right)
\end{array}\right) .
$$


The matrices $P_{i}$ are permutation matrices corresponding to different orderings of the gridpoints.

In [2] it was proved that, for $1 \leqslant k \leqslant 6$, there is a constant $C$, independent of $h$, such that

$$
w^{T} B w>C h^{2} /(\operatorname{diameter}(\Omega))^{2} \cdot w^{T} w,
$$

for all vectors $w$ with dimension equal to the order of $B$. Since $A_{i}$ is a direct sum of matrices of the type $B$, it immediately follows that (2.4) is valid with $B$ replaced by $A_{i}$. It also immediately follows that

$$
v^{T} A v \geqslant n C h^{2} /(\operatorname{diameter}(\Omega))^{2} \cdot v^{T} v,
$$

for all vectors $v$, which implies that

$$
\left\|A^{-1}\right\| \leqslant \frac{(\text { diameter }(\Omega))^{2}}{n C h^{2}},
$$

where the spectral matrix norm has been used. By using this estimate it was proved in [2] that

$$
\begin{gathered}
v=u+h^{2} e_{2}+h^{4} e_{4}+r_{h}, \\
\left\|r_{h}\right\|_{2}=\left(\sum_{x \in \Omega_{h}}\left|r_{h}(x)\right|^{2} h^{n}\right)^{1 / 2}<O\left(h^{5.5}\right),
\end{gathered}
$$

where $e_{2}$ and $e_{4}$ are smooth functions independent of $h$. In order to get a more complete asymptotic expansion for the global error, we need a sharper stability result, with respect to the inhomogeneous term in the boundary condition, than the one that follows from (2.6).

Let $\left[\Omega_{h}^{*}\right]$ denote the set of grid functions $y$ defined on $\Omega_{h}$ with $y(x)=0$ for $x \notin \Omega_{h}^{*}$. We shall now prove that, for $1<k<6$, there is a constant $C_{1}$, independent of $h$, such that

$$
v^{T} A v \geqslant n C_{1} h / \operatorname{diameter}(\Omega) \cdot v^{T} v, \quad \text { for } A v=y \in\left[\Omega_{h}^{*}\right] .
$$

From this estimate it immediately follows that

$$
\left\|A^{-1} y\right\| \leqslant \operatorname{diameter}(\Omega) /\left(n C_{1} h\right) \cdot\|y\|, \quad y \in\left[\Omega_{h}^{*}\right] .
$$

We shall now prove (2.8) by first proving a similar inequality for the matrices of the type $B$. Let us consider the system of linear equations

$$
B w=\left(\begin{array}{c}
g_{0} / \alpha_{0} \\
0 \\
\vdots \\
0 \\
g_{N} / \tilde{\alpha}_{0}
\end{array}\right),
$$

which is a discretization of the one-dimensional problem $-z^{\prime \prime}=0, z(0)=g_{0}$, $z(a)=g_{N}$, where $a$ is a positive constant and $z(x)=g_{0}(a-x) / a+g_{N} x / a$. Let us introduce the gridpoints $x_{\nu}=x_{0}+\nu h, \nu=0,1,2, \ldots, N+1$, where $N$ is the order of the matrix $B$. Further $-x_{0}=s h$ and $x_{N+1}-a=\tilde{s h}$, where $s$ and $\tilde{s}$ are the quantities appearing in $\alpha_{0}$ and $\tilde{\alpha}_{0}$, respectively. The system (2.10) can now be 
written as

$$
\begin{gathered}
-w_{\nu-1}+2 w_{\nu}-w_{\nu+1}=0, \quad \nu=1,2, \ldots, N, \\
-w_{0}=\sum_{j=1}^{k} \beta_{i} w_{j}-\frac{1}{\alpha_{0}} g_{0}, \quad-w_{N+1}=\sum_{j=1}^{k} \tilde{\beta}_{j} w_{N+1-j}-\frac{1}{\tilde{\alpha}_{0}} g_{N} .
\end{gathered}
$$

Since' $z$ is a linear function, $w_{\nu}=z\left(x_{\nu}\right)$, for $k>1$, i.e.

$$
w_{\nu}=g_{0}\left(a-x_{v}\right) / a+g_{N} x_{\nu} / a .
$$

From (2.10) and from the above expression for $w_{v}$, we get

$$
\begin{aligned}
a w^{T} B w & =g_{0}^{2} \frac{a-x_{1}}{\alpha_{0}}+g_{0} g_{N}\left(\frac{x_{1}}{\alpha_{0}}+\frac{a-x_{v}}{\tilde{\alpha}_{0}}\right)+g_{N}^{2} \frac{x_{N}}{\tilde{\alpha}_{0}} \\
& \geqslant g_{0}^{2}\left(\frac{a-x_{1}}{\alpha_{0}}-\frac{1}{2}\left(\frac{x_{1}}{\alpha_{0}}+\frac{a-x_{N}}{\tilde{\alpha}_{0}}\right)\right)+g_{N}^{2}\left(\frac{x_{N}}{\tilde{\alpha}_{0}}-\frac{1}{2}\left(\frac{x_{1}}{\alpha_{0}}+\frac{a-x_{N}}{\tilde{\alpha}_{0}}\right)\right) .
\end{aligned}
$$

Since $a-x_{1}>(N-1) h, x_{N} \geqslant(N-1) h, x_{1}=(1-s) h$, and $a-x_{N}=(1-\tilde{s}) h$ and further $0<\alpha_{0}, \tilde{\alpha}_{0}<1, \alpha_{0} /(1-s)>1 / k$, and $\tilde{\alpha}_{0} /(1-\tilde{s}) \geqslant 1 / k$, we get

$$
w^{T} B w \geqslant \frac{(N-1) h-k h}{a}\left(g_{0}^{2}+g_{N}^{2}\right) \text {. }
$$

Let us now consider the quantity $w^{T} w$ which, according to (2.11), can be written as

$$
\begin{aligned}
w^{T_{w}} & =\frac{1}{h}\left(g_{0}^{2} \sum_{\nu=1}^{N}\left(\frac{a-x_{\nu}}{a}\right)^{2} h+2 g_{0} g_{N} \sum_{\nu=1}^{N} \frac{x_{\nu}}{a}\left(\frac{a-x_{v}}{a}\right) h+g_{N}^{2} \sum_{\nu=1}^{N}\left(\frac{x_{\nu}}{a}\right)^{2} h\right) \\
& <\frac{2}{h} \max \left(\sum_{\nu=1}^{N}\left(\frac{a-x_{\nu}}{a}\right)^{2} h, \sum_{\nu=1}^{N}\left(\frac{x_{v}}{a}\right)^{2} h\right)\left(g_{0}^{2}+g_{N}^{2}\right) \\
& <\frac{2((N+1) h)^{3}}{3 a^{2} h}\left(g_{0}^{2}+g_{N}^{2}\right) .
\end{aligned}
$$

By using (2.12) and the above inequality, we easily get

$$
w^{T} B w \geqslant 3 h(1-(k+2) /(N+1)) /(2 a) \cdot w^{T} w,
$$

where we also have used that $(N+1) h \geqslant a$. For later references we write this inequality as

$$
w^{T} B w>h C_{1} / \operatorname{diameter}(\Omega) \cdot w^{T} w, \quad C_{1}=3 /(2(k+3)), \quad N>k+1 .
$$

Note that (2.13) is valid only for $w$ satisfying (2.10). The inequality (2.8) can now be obtained in the same way as (2.5).

Let us for functions $y \in\left[\Omega_{h}^{*}\right]$ define the following $n$ - 1-dimensional $L_{2}$-norm

$$
|y|_{2}=\left(\sum_{x \in \Omega_{\hbar}^{*}}|y(x)|^{2} h^{n-1}\right)^{1 / 2} .
$$

We can now write (2.9) in the following way

$$
A v=y \in\left[\Omega_{h}^{*}\right] \Rightarrow\|v\|_{2} \leqslant \operatorname{diameter}(\Omega) /\left(n C_{1} \sqrt{h}\right) \cdot|y|_{2},
$$


where $\|\cdot\|_{2}$ is the norm defined in (2.7). For later use we also write down the local truncation error to the extrapolation formula (2.3)

$$
\frac{(-1)^{k}}{k+1} s h^{k+1} u^{(k+1)}
$$

We shall now derive the improved version of the asymptotic expansion of the global discretization error and consider for definiteness the case $k=6$. We make the Ansatz

$$
v=u+h^{2} e_{2}+h^{4} e_{4}+h^{6} e_{6}+r_{h},
$$

where $e_{2}, e_{4}$, and $e_{6}$ are smooth functions, independent of $h$, satisfying the boundary condition $e_{t}=0$, on $\partial \Omega, t=2,4,6$. We shall prove that $\left\|r_{h}\right\|_{2}=O\left(h^{6.5}\right)$. For the solution $u$ of $(2.1)$, we have

$$
A u=h^{2} f+G+h^{4} l_{4}(u)+h^{6} l_{6}(u)+h^{8} l_{8}(u)+O\left(h^{7}\right) G_{1}+O\left(h^{9}\right)
$$

where the $l_{t}$ are differential operators of order $t$ with constant coefficients, $t=2,4$, 6, and $8, G$ and $O\left(h^{7}\right) G_{1}$ belong to [ $\Omega_{h}^{*}$ ] and correspond to the inhomogeneous boundary condition and to (2.15), respectively. We note that the difference scheme is given by $A v=h^{2} f+G$ and further that

$$
A e_{t}=h^{2} L e_{t}+h^{4} l_{4}\left(e_{t}\right)+h^{6} l_{6}\left(e_{t}\right)+O\left(h^{7}\right), \quad t=2,4,6,
$$

where $L$ is the differential operator defined in (2.1). By multiplying the Ansatz for $v$ by $A$ and by using (2.16) and (2.17), we get that

$$
\begin{aligned}
A v= & A u+\sum_{t=1}^{3} h^{2 t} A e_{2 t}+A r_{h} \\
= & h^{2} f+G+h^{4} l_{4}(u)+h^{6} l_{6}(u)+h^{8} l_{8}(u)+O\left(h^{7}\right) G_{1}+O\left(h^{9}\right) \\
& +h^{4} L e_{2}+h^{6} l_{4}\left(e_{2}\right)+h^{8} l_{6}\left(e_{2}\right)+O\left(h^{9}\right)+h^{6} L e_{4}+h^{8} l_{4}\left(e_{4}\right) \\
& +O\left(h^{10}\right)+h^{8} L e_{6}+O\left(h^{10}\right)+A r_{h}=h^{2} f+G .
\end{aligned}
$$

By determining $e_{2}, e_{4}$, and $e_{6}$ by

$$
\begin{gathered}
L e_{2}+l_{4}(u)=0, \quad L e_{4}+l_{4}\left(e_{2}\right)+l_{6}(u)=0, \\
L e_{6}+l_{4}\left(e_{4}\right)+l_{6}\left(e_{2}\right)+l_{8}(u)=0, \quad e_{t}=0 \quad \text { on } \partial \Omega, \quad t=2,4,6,
\end{gathered}
$$

we get

$$
A r_{h}=-G_{1} O\left(h^{7}\right)+O\left(h^{9}\right) .
$$

Since $G_{1} \in\left[\Omega_{h}^{*}\right]$ and $\left|G_{1}\right|_{2}=O(1)$, it follows from (2.14) and (2.6) that

$$
\left\|r_{h}\right\|_{2}=O\left(h^{6.5}\right)
$$

which is the main result of this paper.

Acknowledgements. Thanks are due to Heinz-Otto Kreiss for a number of valuable conversations and for his method for proving the convergence of schemes of the type considered in this paper. I also want to express my indebtedness to the authors of the paper [2]. 
Department of Applied Mathematics

University of Luleà

S-951 87 Luleả, Sweden

1. V. Pereyra, "Accelerating the convergence of discretization algorithms," SIAM J. Numer. Anal., v. 4, 1967, pp. 508-533; MRC Technical Report No. 687, Univ. of Wisconsin, 1966.

2. V. Pereyra, W. Proskurowski \& O. Widlund, "High order fast Laplace solvers for the Dirichlet problem on general regions," Math. Comp., v. 31, 1977, pp. 17-23.

3. G. StaruUs, "Constructing orthogonal curvilinear meshes by solving initial value problems," Numer. Math., v. 28, 1977, pp. 25-48.

4. G. Starius, "Composite mesh difference methods for ellptic boundary value problems," Numer. Math., v. 28, 1977, pp. 243-258. 